ARTÍCULO

\title{
Retos y aprendizajes de la sistematización de experiencias de educación popular. El caso de la Pre-Universidad Tunjuelo Popular en Bogotá*
}

Nicolás Martínez-Gómez'

\section{RESUMEN}

El presente artículo es producto de la investigación realizada alrededor de la sistematización de la experiencia de la Pre-Universidad Tunjuelo Popular en los años 2016 y 2017. Como primera medida, se realiza una caracterización alrededor de la sistematización de experiencias como modalidad investigativa y de la metacognición, para luego profundizar el ejercicio metacognitivo que tiene como objetos de reflexión los resultados de la investigación y el proceso investigativo en sí, señalando algunos retos y aprendizajes de este tipo de investigaciones.

\section{PALABRAS CLAVE}

sistematización; educación popular; metacognición; investigación; organizaciones sociales.

*Esta investigación fue el centro de la tesis de la maestría en estudios sociales de la Universidad Pedagógica Nacional titulada Del río al caracol: transformando la cotidianidad. Sistematización de la experiencia de la Pre-Universidad Tunjuelo Popular (2012-2016). 'Corporación Universitaria Minuto de Dios, Bogotá, Colombia. 


\section{CHALLENGES AND LEARNINGS OF THE SYSTEMATIZATION OF POPULAR EDUCATION EXPERIENCES. THE CASE OF THE TUNJUELO POPULAR PRE-UNIVERSITY IN BOGOTA}

\section{ABSTRACT}

The present article is a product of the investigation around the systematization of the experience of the Tunjuelo Popular Pre-University during 2016 and 2017. As a first measure, a characterization is carried around the systematization of experiences as an investigative modality and of metacognition, in order to delve into the metacognitive exercise whose objects of reflection are the results of the investigation and the investigative process itself, pointing out some of the challenges and learnings of this kind or researches.

\section{KEYWORDS}

systematization; popular education; metacognition; investigation; social organizations.

\section{DESAFIOS E APRENDIZADOS DA SISTEMATIZAÇÃO DE EXPERIÊNCIAS DE EDUCAÇÃO POPULAR. O CASO DA PRÉ-UNIVERSIDADADE POPULAR DO TUNJUELO EM BOGOTÁ}

RESUMO

O presente artigo é produto da investigação realizada sobre a sistematização da experiência da Pré-Universidade Popular do Tunjuelo nos anos 2016 e 2017. Primeiramente, faz-se uma caracterização em torno da sistematização de experiências como modalidade investigativa e da metacognição, para logo aprofundar no exercício metacognitivo que tem como objetos de reflexão os resultados da pesquisa e do mesmo processo investigativo a fim de poder apontar alguns desafios e os aprendizados deste tipo de pesquisas. 


\section{INTRODUCCIÓN}

La educación popular se expresa en prácticas concretas, situadas y contextuadas, y a partir de estas es que la sistematización de experiencias encuentra su lugar al permitir una reflexión y recuperación crítica de los saberes que allí se generan, cuestionan y circulan. La idea que sustenta esta apuesta se basa en que

[...] en la acción existen saberes y de igual manera, en el nudo de relaciones que se construyen, se crean, se procesan y se reconstruyen, y que, a pesar de la minusvaloración que se hace de la práctica, estos generan saber, conocimiento, sabiduría, emociones, sentidos, apuestas políticas y éticas, y que cuando se hace el trabajo de visibilizarlos, no son formas minoritarias del conocimiento científico, ya que lo conciben a este, se diferencian y muestran su nudo de relaciones. (Revista Internacional Magisterio, 2008, p. 6)

Sin embargo, los saberes surgidos en la práctica por sí mismos no generan nuevo conocimiento. Sí lo hacen el análisis y la interpretación de estos saberes "preexistentes" confrontados con la realidad y, por tanto, a partir de las reflexiones (sistematizaciones) de estas experiencias. Por eso se ubica allí un tránsito de saberes a conocimientos, en el sentido que se va de los saberes "implícitos, difusos, imprecisos, no verbalizados ni argumentados, a los conocimientos explícitos, delimitados, precisos, verbalizados y argumentados" (Vasco, 2008, p. 21).

Otro elemento particular lo señala Alfredo Ghiso (1998,p.4) cuando afirma que

Tanto prácticas como sistematizaciones asumen marcos referenciales, direccionalidades y procesos operativos de acuerdo con los sujetos: minorías étnicas, campesinos desplazados por la violencia, culturas juveniles, grupos de mujeres, niños de la calle, reinsertos a la vida civil, organización de venteros ambulantes, movimientos ecológicos, grupos culturales. Cada sector va desarrollando su práctica, la reflexiona y toma la palabra para trasmitir saberes que habían sido silenciados por discursos homogeneizadores e invisibilizadores de la diversidad y de los múltiples contextos en la que heterogeneidad económica, política, ecológica, social y cultural se soportan y de las relaciones que, entre alteridades, se establecen para legitimar, circular, hacer uso y apropiar conocimientos para la acción social.

Así entonces, las sistematizaciones de experiencias responden a una pluralidad de factores que surgen de prácticas contextuadas y situadas en realidades particulares. Debido a esto no existe un consenso entre las distintas concepciones frente a esta "práctica intencionada de generación de nuevo conocimiento sobre las prácticas educativas y sociales"(Mendoza y Torres, 2013, p. 165). Se podría afirmar que habría tantas sistematizaciones como prácticas, pues las primeras responden a las particularidades de las segundas, así como al lugar de enunciación y punto de vista de quienes las investigan. Además, las justificaciones para desarrollar sistematizaciones, las perspectivas epistemológicas desde donde se fundamentan, y las maneras de operativizarlas metodológicamente varían ampliamente. 
Por otra parte, si bien es importante la producción, cualificación y visibilización de estos conocimientos, también lo es el análisis del proceso investigativo en sí mismo, pues allí se generan aprendizajes y aportes a la sistematización de experiencias como modalidad investigativa. Me refiero al momento de la metacognición, que adquiere especial relevancia en la medida en que las investigaciones no solo no son neutrales ni objetivas, sino que nunca están exentas de tensiones, errores o conflictos. Este ejercicio metacognitivo expresa la reflexión sobre el cómo se desarrolló la investigación que se pueden potenciar los aprendizajes para cualificar la realización de sistematizaciones de experiencias.

Este tipo de investigaciones están atravesadas por los intereses de quienes participan en ellas, tanto los propios protagonistas de la organización social, como los asesores y asesoras externas provenientes de la academia. Por ejemplo, en la presente investigación mi rol no fue solo de investigador sino también de integrante activo ${ }^{1}$ por lo que las apuestas políticas y educativas de la Pre-Universidad Tunjuelo Popular, ${ }^{2}$ al ser compartidas por mí, entran a jugar en cómo me sitúo como sujeto y como investigador.

Como parte de la expresión de esta mixtura de intereses, en la escritura se pueden presentar ciertas desviaciones que, si bien "no son, por fortuna, una constante existente en todas las sistematizaciones; son más bien, 'peligros latentes' que por eso mismo es positivo tenerlos presentes" (Mariño, 2004, p. 40). Entre estas desviaciones están las verdades a medias, que operan bajo la lógica de no decir mentiras, pero no decir toda la verdad y se relaciona con la necesidad de mantener la financiación de alguna entidad, intentando mostrar solo la cara bonita de los procesos. También se puede maquillar la información para minimizar o no visibilizar defectos, dificultades, contradicciones o problemas, y más bien construyendo una historia oficial armónica y sin sobresaltos. Esto último se relaciona con una especie de narcicismo en el que lo importante es cuidar la imagen de la organización y donde se proyecta una imagen idealizada hacia el exterior. Otro elemento que problematiza éticamente la investigación es cuando surgen datos que corresponden al ámbito privado de las personas, por ejemplo, en términos de amores u odios que emergen al interior de la organización social.

Es necesario visibilizar y analizar estos elementos pues, si bien pueden afectar la transparencia de la sistematización, esto hace parte de un ejercicio de responsabilidad de quienes investigan, de dar cuenta no solo de los resultados sino del proceso y la forma en la que se tomaron decisiones o se resolvieron conflictos, sus dificultades y sus cambios. Este ejercicio desidealiza el proceso investigativo y lo somete a reflexión, no solo para validar su ejecución y analizar epistemológica y metodológicamente lo investigado, sino para posibilitar que otras experiencias que quieran sistematizar su proceso aprendan tanto de los aciertos como de los errores que se presentaron.

Así entonces, el presente artículo tiene como objetivo profundizar el ejercicio metacognitivo de la sistematización de la experiencia de la Pre-Universidad Tunjuelo Popular, en calidad de tesis de la maestría en estudios sociales de la Universidad Pedagógica Nacional. Se someterá a análisis el proceso de investigación en sí, tomado como objeto de estudio, explicitando los cambios ocurridos, así como los aciertos y dificultades identificadas, para contribuir a la cualificación de pesquisas similares.

1 Estoy vinculado a Tunjuelo Popular desde septiembre de 2015.

2 Colectivo de educación popular nacido en Bogotá en 2012. 
Para esto, se caracterizará inicialmente la sistematización de experiencias como modalidad investigativa y la metacognición del proceso investigativo.

\section{CARACTERIZANDO LA SISTEMATIZACIÓN DE EXPERIENCIAS}

La sistematización de la experiencia de la Pre-Universidad Tunjuelo Popular se posicionó desde la apuesta de Mendoza y Torres (2013): una perspectiva interpretativa crítica que en términos metodológicos se traduce en una investigación cualitativa y participativa. Esta implica una posición crítica frente a la distinción sujeto/objeto de conocimiento, una intencionalidad transformadora, una posición de los sectores o sujetos investigados/subordinados como sujetos o actores activos y productores de conocimiento (y no como simples fuentes de información), entre otros. Así, su postura epistemológica se desarrolla a partir de los cinco siguientes elementos:

1. Producción de conocimiento desde los sujetos de la experiencia. En contraposición a la perspectiva de que quien investiga es un observador externo a su objeto de estudio para así garantizar objetividad, desde esta perspectiva se ve la sistematización como un sistema auto-observador en donde los actores/observadores problematizan su realidad a través del diálogo con otros actores de la experiencia. Se desplaza así el principio de objetividad por el de reflexividad, donde se dialoga sobre los alcances y límites de su posición de observadores, de sus propias observaciones y de los objetos de conocimiento que construyen.

2. Noción de la realidad y papel de la ciencia social. Se asume que quién investiga no parte de cero y en cambio incorpora y pone en juego su corriente de pensamiento, su disciplina de conocimiento, su sistema cultural, entre otros. Además, para esta perspectiva crítica, la realidad (y su percepción) está atravesada por relaciones de poder, por lo que no habría lecturas neutrales de la misma: "la preocupación de las ciencias sociales será entonces, comprender dichas realidades desde el marco de referencia de las coordenadas culturales y políticas en las que se desenvuelven los actores" (Mendoza y Torres, 2013, p. 163).

3. Estrategias de abordaje del problema. Aquí se combinan diversas modalidades de acercamiento a los objetos de análisis para captarlos en su complejidad como síntesis de múltiples relaciones y potencialidades, con el fin de reconocer y comprender las maneras como los sujetos se representan, interactúan y construyen realidades sociales específicas.

4. Papel de quienes investigan y su relación con los sujetos. Quien investiga se involucra activamente, en un movimiento de formación y transformación conjunta con el grupo social que investiga.

5. La sistematización como reconstrucción de sentidos de la experiencia. Las prácticas sociales y educativas a sistematizar se conciben como realidades complejas, como construcciones históricas y de sentido donde confluyen factores y dinámicas objetivadas con procesos e interacciones subjetivas, que además están atravesadas por relaciones de poder. La sistematización busca dar cuenta de esa interacción entre contextos, prácticas y sentidos. 
Esta investigación planteó un enfoque metodológico de tipo cualitativo-participativo, partiendo de una actitud de apertura en términos de la especificidad de la experiencia organizativa estudiada, así como la posibilidad de que a lo largo del proceso las preguntas de investigación inicialmente planteadas pudieran cambiar, o que los hallazgos y avances del propio proceso investigativo dieran paso a la necesidad de explorar nuevas elaboraciones metodológicas y conceptuales. Si bien se parte de una experiencia concreta de educación popular, hay que recordar que "los estudios cualitativos generan conocimientos que eventualmente pueden cobijar a muchos casos sin que pretendan tener un carácter universal e independiente de los contextos y de las épocas" (Mariño, 2011, p. 132).

Luego de puntualizar algunas características particulares de la sistematización, a continuación, mencionaré los cuatro grandes momentos que, en general, se presentan en una sistematización de experiencias:

1. Discusión inicial y diseño global de la propuesta. Se dialoga alrededor de la propuesta de sistematización y definen los objetivos, criterios y finalidades del proceso con los y las integrantes de la experiencia a sistematizar. Se llega a un acuerdo frente a la forma en que se va a desarrollar el proceso y las responsabilidades que se van a asumir frente al mismo.

2. Reconstrucción oral de la trayectoria bistórica de la experiencia. Teniendo en cuenta los antecedentes y contexto de surgimiento de la experiencia que se va a sistematizar para poder situarla, se precisan las preguntas y temáticas sobre las cuales se organizará la reconstrucción descriptiva de la experiencia.

3. Análisis e interpretación conjunta de la experiencia. Este momento de la investigación se puede dividir en dos: el análisis y la interpretación. E1 momento de análisis representaría un primer momento de abstracción de la práctica al filtrarla y decantarla en unas categorías útiles para su análisis y que den cuenta de los nodos temáticos alrededor de los cuales se quiere profundizar. Allí, se realiza una lectura transversal de la reconstrucción de la experiencia y se identifican los tópicos recurrentes que aparezcan como significativos, que se convertirán en los ejes temáticos alrededor de los cuales se organizará la información. En el momento de interpretación, según Mendoza y Torres (2013), se hace uso de los referentes teóricos que surjan en el desarrollo de la investigación para construir nuevas lecturas explicativas y comprensivas de la experiencia, donde se evidencie la lógica que la ha configurado para posibilitar una nueva legibilidad de la cual puedan derivar decisiones para cualificar la práctica.

4. Momento prospectivo. Una sistematización tiene la intención de generar una apropiación analítica y crítica de la experiencia por sus propios protagonistas, para así generar cambios en las formas en las que se ha venido actuando y pensando. En este momento se da una reflexión crítica de la experiencia hacia el pasado, para plantear líneas de trabajo hacia el futuro que aprovechen los aprendizajes derivados del proceso de sistematización. 


\section{LA METACOGNICIÓN}

La metacognición corresponde al ejercicio de sistematizar la sistematización. Es decir, se espera reconstruir analíticamente la manera como se desarrolló el proceso investigativo y reflexionar sobre sus distintos momentos, identificando fortalezas, debilidades y explicitando los cambios que se dieron en el camino, para así también ubicar los aprendizajes y tensiones que pudieron surgir. Esto, porque no es "suficiente con haber participado en la sistematización, había que comprender cómo se hizo; cómo se había producido conocimiento sobre la experiencia" (Cendales, 2004, p. 113), sirve para ver cómo se hizo lo que se hizo, cómo se analizó y cómo se interpretó la experiencia. Es un esfuerzo que contribuye a fortalecer la teorización en relación con esta modalidad investigativa y permite a los grupos cuya experiencia es objeto de estudio, "no solo 'hacer', sino ‘saber cómo se hace"' (Mariño, 2011, p. 12).

Es un momento de reflexión sobre el camino metodológico recorrido que permite trascender la propia experiencia de la sistematización, y a su vez posibilita que quienes participaron en el proceso puedan apropiarse de herramientas metodológicas y teóricas que puedan ayudar a la realización de otras sistematizaciones particulares. Como menciona Lola Cendales (2004, p. 113):

La reconstrucción analítica del proceso es un ejercicio de metacognición que viene a complementar el proceso formativo que se instala en todo el desarrollo de la sistematización; en la explicitación de la propia experiencia, en la contrastación de conceptos y de prácticas, en la transferencia de los talleres a la sistematización del proyecto, en la recapitulación que se hace en cada uno de los eventos, etc. La metacognición es la posibilidad de aprehender y aprender del proceso vivido; es la posibilidad de recrear y trascender la propia experiencia de sistematización.

El ejercicio metacognitivo desarrollado se propone como un modesto intento de confrontación de saberes, en la medida en que hace parte del juego de enfrentar y superar las exclusiones epistemológicas que han sufrido los saberes otros. Esas exclusiones en las que

lo otro es negado a nombre de lo universal, los saberes a nombre de la ciencia, lo comunitario a nombre del individuo y lo personal, lo multicultural a nombre de lo monocultural, los sentidos a nombre de la razón y muchos otros dualismos que el poder ha instituido para negar o invisibilizar lo otro y forjar redes invisibles de interlocución y negación. (Mejía, 2016, p. 246)

Esta confrontación de saberes se relaciona con colocar con fuerza propia, desde sus narrativas y epistemes, aquellos saberes negados o invisibilizados, "haciendo visible su propio rigor y trazando puentes para optimizar y fundamentar las múltiples diversidades"(Mejía,2016, p. 247). Es decir, hace parte de la construcción de un estatuto propio desde las prácticas y conocimientos de lo subalterno.

En este sentido, como menciona Mariño (2011), en la metacognición no sólo entra a jugar una lectura analítica sino también socio-afectiva, confrontando 
así las visiones técnicas y sin vida en las que se suelen expresar las investigaciones en ámbitos académicos. Los ejercicios investigativos realizados en contextos sociales, realizados por y con personas, están atravesados no solo por la racionalidad sino también por la emocionalidad, y en esa medida los análisis e interpretaciones pasan no solo por la cabeza sino también por el corazón.

Este ejercicio reflexivo adquiere una especial importancia en investigaciones que se ubican desde una perspectiva crítica pues estas

[...] no se agotan en la producción de conocimiento; dado que su punto de partida y de llegada es la práctica misma, su reconstrucción narrativa, analítica e interpretativa cobra sentido en la medida en que contribuya a mejorar, a transformar las propias experiencias estudiadas. Esta relación entre conocimiento y acción es vital para las organizaciones, pero no se da en forma mecánica. (Torres, 2011, p. 53)

Las reflexiones sobre la práctica investigativa como la que acá se propone, se posicionan epistemológicamente desde una perspectiva crítica en dos sentidos, y desde allí adquieren una relevancia particular en términos de problematizar la necesidad de no reducir el conocimiento producido a círculos académicos, sino que sean material de debate y formación para las organizaciones sociales.

Por un lado, se expresa una pretensión crítica de la ciencia en la medida en que se asume que esta está revisando constantemente los criterios de su propia validez y que está abierta a los cambios que de allí se deriven. De acuerdo con esta pretensión crítica

[...] los conceptos, hipótesis, explicaciones, comprensiones o métodos científicos son, por su propia naturaleza, temporales y relativos, o absolutos solo con referencia a la temporalidad en la que son validados por la comunidad científica, o al menos por las comunidades dominantes dentro del campo social de la ciencia, de conformidad con los parámetros establecidos por la razón. (Múnera, 2009, p. 17)

De allí que este juicio racional sobre la validez epistemológica de un saber permite al mismo tiempo delimitar el conocimiento científico del no-científico y definir la ciencia como sistema abierto a su propia transformación. Es decir, los ejercicios metacognitivos posibilitan una reflexión autocrítica de la forma en la que se han producido conocimientos en una investigación.

Por otro lado, la posición crítica se expresa en la medida en que visibiliza el lugar y la posición valorativa desde donde se construye el conocimiento científico. Es decir, visibiliza su carácter social, pues toma como punto de partida

la experiencia de dominación, sujeción o explotación de quienes son objetos de conocimiento. El lugar de los otros pasa a ser también el lugar de la crítica. La emancipación de quienes materialmente y en términos concretos no pueden acceder a la racionalidad prometida por la ciencia, se convierte en la finalidad de la crítica misma. (Múnera, 2009, p. 16) 
Es así que ese carácter de lo otro no es solo un lugar "sino una forma de describir, comprender, explicar e interpretar el mundo social desde las racionalidades que en medio de las relaciones de poder, en las que también están inmersas las ciencias, han sido invisibilizadas u ocultadas" (Múnera, 2009, p. 16-17). Esta posición crítica entonces toma otra tonalidad en la medida en que cuestiona permanentemente a la ciencia como manifestación del saber-poder problematizando y transformando la racionalidad científica hegemónica.

Así entonces, me sumo al llamado realizado por Marco Raúl Mejía (2016) en torno a la apuesta por realizar una producción de conocimiento situada, que reconozca la pluriversidad (no eurocéntrica) que renuncia a colocarse en un marco cultural diferente al que tenemos desde nuestras raíces más profundas, desde el sur, desde Latinoamérica.

Es allí donde se encuentra un eco en Orlando Fals Borda en la búsqueda por construir convergencias entre el pensamiento popular y la ciencia académica a partir de un rescate crítico de lo popular, recordando que "el conocimiento popular siempre ha sido fuente del conocimiento formal" (Fals Borda, 1999, p. 76). Como menciona Múnera (2009), la necesidad de valorar los saberes populares implica que se construya un reconocimiento de sus formas de conocimiento, sus experiencias de vida y su cultura para que tengan garantías para su pleno desarrollo y para establecer un diálogo con las ciencias sociales que permita la transformación mutua; es decir, que no sean relegadas a un plano secundario, a un lugar subalterno y precientifico.

En últimas, este ejercicio de reflexionar sobre la propia práctica investigativa en clave crítica se relaciona con la necesidad de cuestionar la idea de que hay una forma única de conocimiento válido, $\mathrm{y}$ reconocer la diversidad de formas de conocimiento, que son tantas cuantas sean las prácticas sociales que las generan y sostienen. Es así que "prácticas sociales alternativas generan formas alternativas de conocimiento. No reconocer esas formas del conocimiento, implica deslegitimar las prácticas sociales que las apoyan y, en ese sentido, promover la exclusión social de los que las promueven" (De Sousa Santos, 1998, p. 491, citado en Múnera, 2009).

\section{SISTEMATIZANDO NUESTRA SISTEMATIZACIÓN}

Durante los años 2016 y 2017 realicé la sistematización de la experiencia de la Pre-Universidad Tunjuelo Popular (Martínez-Gómez, 2017), cuyas dificultades y aprendizajes a continuación abordaré. Para tal propósito se realizará la reconstrucción analítica del proceso.

En primera instancia, es necesario decir que la Pre-Universidad Tunjuelo Popular es un colectivo que, tomando del río Tunjuelo ${ }^{3}$ su nombre, nace en el 2012 en Bogotá a partir del interés de varias personas ${ }^{4}$ por generar espacios educativos

3 El río Tunjuelo atraviesa la mayoría de las localidades (subdivisiones administrativas) del sur de Bogotá, donde se encuentra la mayoría de sectores populares, y por eso se tomó como referente identitario.

4 La mayoría de los integrantes iniciales eran estudiantes de la Universidad Nacional de Colombia y algunas tenían experiencia organizativa previa. 
transformadores y por contribuir a la reducción de la desigualdad frente al acceso a la educación superior por parte de sectores populares. ${ }^{5}$ De allí que su principal práctica haya sido el desarrollo de un preuniversitario popular y gratuito ${ }^{6}$ donde las personas que integran el colectivo hacen las veces de educadoras/talleristas abordando las distintas áreas del conocimiento que evalúan las pruebas de acceso a la educación superior de las universidades públicas de Bogotá y las Pruebas Saber $11^{7}$ del Instituto Colombiano para la Evaluación de la Educación (ICFES). En los distintos ciclos del preuniversitario han trabajado componentes como razonamiento cuantitativo, lectura crítica, pensamiento social nuestro-americano o talleres sobre teatro, género, agro-eco-biología, entre otros. Desde su inicio ha estado articulado con la Coordinadora de Procesos de Educación Popular en Lucha (CPEPEL) que también nace en el año 2012 y articula en el momento (mediados de 2018) a siete colectivos $^{8}$ que trabajan desde la educación popular en Bogotá. Desde febrero de 2016, en conjunto con el Grupo Libertario Vía Libre, ${ }^{9}$ se tiene una casa en arriendo que se ha nombrado Rincón Cultural El Caracol y que hace las veces de sede de Tunjuelo Popular.

La sistematización de esta experiencia se desarrolló a partir de cinco momentos. En el primer, se realizó la socialización de la propuesta para llegar a acuerdos básicos del proceso de investigación. En segundo momento, se reconstruyó descriptiva y narrativamente la trayectoria histórica de la experiencia para identificar los distintos periodos que ha atravesado desde su inicio hasta cuando se realiza la sistematización. Esto, a través de un ejercicio de cartografía social con actuales integrantes de Tunjuelo Popular.

El tercer momento de la investigación se dividió en dos. Por un lado, se analiza la experiencia identificando los núcleos temáticos relevantes para organizar la información alrededor de ellos. Allí, se ubicaron dos grandes polos: la apuesta política y la apuesta educativa. Se utilizaron entrevistas semiestructuradas a miembros de Tunjuelo y a ex educandas, y se realizaron tres grupos focales con

5 En Colombia el acceso a la educación superior, especialmente a la educación superior pública, está sometido a exámenes de selección que generan tasas de admisión muy reducidas (por ejemplo, la tasa de la Universidad Nacional de Colombia admite es de solo al 8\% según Mantilla, 2016).

6 Un preuniversitario es un curso preparatorio para las pruebas de admisión a universidades públicas, así como para las Pruebas Saber 11. Como los resultados de las pruebas Saber 11 son en muchos casos definitivos para acceder a la educación superior, existe una oferta de cursos preparatorios de carácter privado. La intención de ofrecer un preuniversitario gratuito (ofertado por organizaciones sociales y colectivos) es que los sectores populares no dependan de si disponen de la capacidad económica para acceder a estos cursos.

7 Estas pruebas las realizan los y las estudiantes de último grado en el colegio y son un requisito para poder recibir el grado de bachiller. Los resultados de estas pruebas son usados por muchas universidades para definir la admisión a sus programas.

8 Además de Tunjuelo Popular, está el Pre-ICFES Popular del Centro, el Colectivo También el Viento, el Colectivo de Educación Popular Siembra, la Colectiva Vida y Letra, el Colectivo de Acción Popular (CLAP) y el Pre-ICFES Popular de Suba.

9 El Grupo Libertario Vía Libre se autodefine como una agrupación política revolucionaria de tendencia comunista libertaria que nace en junio de 2010 en Bogotá. 
actuales miembros de Tunjuelo. Por otro lado, se interpretó la experiencia a partir de herramientas teóricas que permitieran construir nuevas lecturas explicativas y comprensivas de la experiencia, donde se evidenciara la lógica que la ha configurado.

El cuarto momento, prospectivo, se refiere a cuando la misma organización, producto de los aprendizajes derivados del proceso de sistematización se plantea líneas de acción futuras para poder cualificar su propia experiencia.

El quinto momento, la metacognición, consiste en una reflexión sobre el camino metodológico recorrido que permite trascender la propia experiencia de la sistematización como se mencionó anteriormente. Es sobre este último momento que se hará especial énfasis en el artículo.

\section{SOCIALIZACIÓN DE LA PROPUESTA Y RECONSTRUCCIÓN DOCUMENTAL DE LA EXPERIENCIA}

La explicitación de la propuesta y la intención de sistematizar la experiencia se hizo en febrero de 2016, luego de haber participado en la asamblea semestral en enero de ese año. Esto respondió a que se pudo identificar que desde el comienzo de las actividades en 2012 se tenía la intención de adelantar un proceso de sistematización, pero nunca se había dedicado el tiempo necesario para desarrollar un ejercicio investigativo de este tipo. Además, el hecho de que en 2016 se cumplieran cinco años del proceso también marcaba un momento propicio para realizar un balance de lo realizado. Esto es importante señalarlo pues una sistematización de experiencias no puede surgir exclusivamente del interés de quien investiga por realizarla, sino que primordialmente tiene que responder de una intención y una necesidad de la misma experiencia organizativa. Es decir, no puede ser un ejercicio impuesto o forzado.

A partir de la socialización de la propuesta, se conformó un comité de sistematización que sería el encargado de desarrollar la investigación en la medida en que las personas que conformaran ese comité lo quisieran hacer, es decir, no tenían que desarrollar todas las actividades que surgieran sino sólo con las que se pudieran comprometer. Así, inicialmente este comité estuvo conformado por cinco personas del proceso.

$\mathrm{Al}$ respecto, es pertinente lo que menciona Alfonso Torres frente al desafío que en las sistematizaciones implica la formación de los sujetos de la investigación, pues "a diferencia de la investigación clásica, en la que se supone que los investigadores 'ya están formados'y sus roles están predefinidos, en la perspectiva interpretativa crítica está la preocupación permanente sobre cómo se conforma el sujeto colectivo del estudio" (Torres, 2011, p. 52). En nuestro caso, no se presentó la dicotomía entre académicos y activistas sociales, entre agentes externos y sujetos de base pues quien actuaba desde el rol de investigador era al mismo tiempo parte de la experiencia organizativa, y las demás personas del colectivo tenían formación universitaria. Esto provocó, como menciona también Torres (2011), que hubiera investigadores-actores sociales y se combinaran posiciones tanto académicas como prácticas.

Uno de los primeros cambios que ocurrió se relaciona con un momento investigativo que se había propuesto inicialmente: la reconstrucción documental 
de la experiencia. La primera tarea que se acordó fue realizar la revisión del correo electrónico de Tunjuelo Popular intentando rastrear documentos que posibilitaran identificar hechos o momentos significativos que permitieran ir reconstruyendo históricamente la experiencia. En el camino, una persona del comité manifestó que debido a su disponibilidad de tiempo no podía seguir aportando al proceso de sistematización y otra más se alejó tanto del Comité como de Tunjuelo en general. Debido a esto, sumado a que la tarea implicaba un nivel significativo de trabajo que nos habíamos propuesto realizar en un plazo irreal de dos semanas -, se decidió desistir y seguir con el siguiente momento de la investigación: la reconstrucción oral de la trayectoria histórica de la experiencia.

\section{LA RECONSTRUCCIÓN ORAL DE LA TRAYECTORIA HISTÓRICA DE LA EXPERIENCIA}

En este momento el equipo encargado de la sistematización se había reducido a tres personas, y se tenían proyectadas varias actividades a realizar: el taller de cartografía social (que no estaba previsto, pero emergió como útil al momento de desarrollar la recolección de la información), grupos focales y entrevistas individuales no solo con miembros actuales de Tunjuelo sino también con ex integrantes y ex educandos o educandas. A propósito, cabe señalar que el taller de cartografía social fue un acierto en la medida en que se generó una espacialización de la trayectoria de la experiencia, permitiendo que esta se encarnara en una representación gráfica del territorio en el que habita.

Si bien la propuesta y el diseño de los grupos focales y las entrevistas era responsabilidad mía, la intención era que las entrevistas no las realizara sólo yo, no sólo para distribuir el trabajo de la investigación, sino con la intención de que el saber-hacer investigativo no se concentrara en una sola persona y que, en cambio, fuera un ejercicio y un aprendizaje colectivo. Estas tareas las asumieron quienes estaban en el comité de sistematización, que ya mostraba su inoperancia y terminaría por desintegrarse prematuramente.

Durante el proceso de recolección de la información, específicamente a partir de los grupos focales y las entrevistas individuales, fue necesario tomar dos decisiones frente al desarrollo de la investigación.

La primera, se relaciona con que inicialmente se habían propuesto cuatro posibles ejes (relacional, identitario, político y educativo) sobre los cuales indagar y que sirvieran como categorías analíticas para organizar la información. Sin embargo, no había mucha claridad en las y los integrantes de Tunjuelo sobre los ejes relacional e identitario, por lo que se tomó la decisión de reducir los ejes de cuatro a dos y solo concentrarse en la apuesta política y en la apuesta educativa de Tunjuelo.

La otra decisión que se tuvo que tomar fue frente a la pregunta de cuándo parar. Inicialmente se había pensado tomar como momento de corte julio de 2016 porque era el fin de las sesiones del preuniversitario. Sin embargo, en el segundo semestre de 2016 se realizaron varios eventos (conciertos y fiestas) y se desarrollaron talleres libres en el Rincón Cultural El Caracol (danza andina, portugués, 
artesanías y tejido, escrituras creativas, fitness y acondicionamiento físico, arte y literatura para niños y niñas, fotografía, artesanía para niños y niñas y cine-foros), por lo que se consideró importante ampliar el periodo estudiado y pasar el corte a diciembre de 2016.

Este momento de la investigación permitió establecer momentos de auge donde las y los integrantes de Tunjuelo Popular presentaban un nivel alto de compromiso (2012-2013), así como un momento de crisis (2014-2015) donde se genera un desgaste expresado por ejemplo un alto número de horas de clase por persona, en la falta de autocrítica (el sisiseverismo), ${ }^{10}$ en la falta de espacios de encuentro entre talleristas, en tensiones al interior del proceso, en el retiro de varios y varias talleristas comprometidas y la consiguiente concentración de tareas en pocas personas.

Un elemento presente durante los primeros cuatro años de existencia era el constante traslado de la sede de las actividades del preuniversitario, lo que incidió en la misma estabilidad del colectivo. A partir del 2016 con el Rincón Cultural El Caracol, se puede pensar en una proyección de las actividades de Tunjuelo en un mediano plazo y con un anclaje territorial.

Como un ejercicio de autocrítica, es importante señalar que en este tipo de procesos investigativos se debe propiciar e impulsar la participación como una tarea vital y de constante trabajo, y no como un elemento que se asuma de antemano. Como señala Torres (2011, p. 52):

la participación no es una práctica natural, sino que hay que construirla, posibilitando condiciones para que las personas que se involucren en el proyecto fortalezcan su capacidad de tomar decisiones a lo largo del proceso y asuman las responsabilidades que se deriven de él.

En este caso, al ver que el comité de sistematización no funcionaba se debió proponer, en ese mismo instante, una alternativa para reactivar la participación de los y las integrantes de Tunjuelo y no simplemente esperar a que se vincularan al ejercicio investigativo por pura fuerza de voluntad. El mismo Alfonso Torres (2011) señala la importancia de gestar espacios formativos, especialmente talleres alrededor de las metodologías y estrategias involucradas. Y específicamente allí se identifica un error en la medida en que faltó un escenario previo de formación alrededor de la sistematización de experiencias, sus objetivos, la importancia de que fuera un ejercicio colectivo y el impacto en el futuro del colectivo. Esto con la intención de que el proceso investigativo hubiera tenido un carácter realmente participativo y se hubiera podido generar una producción de conocimiento colectiva, en la que las personas que integran Tunjuelo actuaran en calidad de sujetos y sujetas, y no sólo como objetos de investigación.

Este elemento es de vital importancia, como me lo señaló Santiago Gómez (2017, comunicación personal):

10 Esta expresión hace referencia a que en los momentos de autocrítica o en los momentos decisorios en los que se consultaba algo a los y las integrantes, la respuesta más común era "si, si, severo". Es decir, no se expresaban propuestas ni se manifestaban desacuerdos sino simplemente se asumía lo que decidiera el resto. 
el carácter participativo de la sistematización de experiencias desde un enfoque de Educación Popular, es uno de los elementos centrales y vitales de esta apuesta investigativa. De lo contrario, su pretensión e intencionalidad formativa-transformadora (uno de los elementos más decisivos e importantes que justifican la operacionalización del diálogo educativo, a partir de la mediación necesaria de la investigación en el proceso de aprendizaje) queda profundamente minada y/o desvirtuada, toda vez que las capacidades investigativas, pedagógicas, conceptuales y metodológicas de las personas, organizaciones e instituciones involucradas, no se ven radicalmente afectadas en el proceso de reflexión colectiva y apropiación sistemática de la experiencia por parte de sus propias y propios integrantes.

Lo anterior se relaciona con el proceso de retroalimentación y discusión colectiva de los documentos que surgieran de la sistematización. Frente a las distintas versiones del escrito producido hubo algunas correcciones de integrantes del proceso, pero la intención de que el proceso de investigación fuera colectivo ya empezaba a verse debilitada por la inasistencia de la mayoría de los integrantes a los grupos focales.

La poca participación en este momento de la investigación no es un elemento para tomar a la ligera pues representaba la posibilidad de contar y contarse, de generar un escenario de diálogo entre las personas "antiguas" y las personas "nuevas" del colectivo, de poder enterarse de actividades o acontecimientos en las que no se había participado, de reconocer los sentimientos y afectos que atravesaban su participación en la organización, y permitir la reflexión sobre la trayectoria de la experiencia a partir de la remembranza de lo vivido y sentido en la propia práctica. Este momento narrativo adquiría una especial importancia debido a que el relato abre

la posibilidad de liberar la experiencia única e irrepetible; es la posibilidad del sujeto de construir su realidad y de configurar su propia identidad. Siendo personal es también intersubjetivo, lo cual posibilita la construcción colectiva de realidad y la posibilidad de imaginarse visiones de futuro y utopías sociales. Es una posibilidad de incluir a los otros en el recuerdo, la resistencia y en los procesos de transformación. (Cendales y Torres, 2006, p. 38)

En retrospectiva, algo que hubiera sido importante realizar para facilitar la circulación del relato que se estaba construyendo era imprimir un librito o cartilla que fuera objeto de lectura y diálogo colectivo. Esto demostró buenos resultados en otras sistematizaciones como las realizadas por Gómez, Medina y Riaño (2012).

\section{EL MOMENTO ANALÍTICO E INTERPRETATIVO DE LA EXPERIENCIA}

Frente al análisis de la experiencia continuó la dinámica de baja participación. En los grupos focales en los que se profundizaba sobre la apuesta política y educativa de Tunjuelo Popular la asistencia seguía siendo baja. A pesar de que se intentó que el comité de sistematización funcionara de nuevo, las tareas de la investigación se concentraron en mí. Si bien se intentó que otros integrantes realizaran algunas 
entrevistas (que no se realizaron) y las transcribieran, tanto el diseño de los grupos focales, como la grabación de lo que se discutía, la transcripción de los audios y la redacción de los documentos síntesis fue tarea exclusiva mía.

Alrededor de la apuesta política se identificaron algunos principios políticos que entraban dentro de un consenso amplio (principio anticapitalista, principio antipatriarcal, ecologismo y diversidad orgánica) y otros principios presentes desde una posición periférica (antiespecismo, vegetarianismo, decolonialismo, anarquismos, autonomismos y poder popular). Se identificó así mismo la horizontalidad como principio organizativo que implica la intención de que las decisiones no se concentren en pocas personas y que no existan instancias jerárquicas, sino que estas se tomen basadas en el diálogo y el consenso. Se hizo explícita también la intención de ir más allá de la práctica educativa y desarrollar un trabajo que "echara raíces" en el territorio.

Frente a la apuesta educativa, ésta se ubicó desde la educación popular ${ }^{11}$ materializada a través del desarrollo de un preuniversitario popular y gratuito con jóvenes, en el que se puede identificar una tensión entre lo urgente, es decir,los temas que se les van a evaluar en la Prueba Saber 11 del ICFES - cuyo abordaje es necesario para que puedan sacar mejores resultados y así poder acceder a la educación superior -; y lo fundamental, es decir, los intereses y las apuestas del colectivo para que a través de la práctica educativa se puedan generar herramientas para leer el mundo desde una perspectiva crítica y transformadora.

Cuando se consideró que había suficiente información en el momento analítico como para pasar al momento interpretativo, se inició la búsqueda de las herramientas teóricas que sirvieran para comprender e interpretar la experiencia. Esta tarea sería mi responsabilidad y se vería expresada en dos textos (uno sobre la apuesta política y el otro sobre la apuesta educativa) que se compartieron con las personas integrantes de Tunjuelo.

En la asamblea semestral de Tunjuelo, realizada en diciembre de 2016 se dio la oportunidad de abordar el tema específico de la sistematización. Allí se realizó la socialización de lo que se había realizado hasta entonces y se quería dialogar acerca de las dificultades que se habían presentado. Por parte del resto de integrantes de Tunjuelo se aceptó que efectivamente no hubo mucho apoyo frente al proceso de sistematización y las tareas se habían concentrado, a pesar de que se había llegado a un acuerdo inicial de que el proceso iba a ser colectivo. Frente a esto se propuso que, como contrapartida o aporte del resto de Tunjuelo a la sistematización, se realizara una cartilla donde se resumiera la investigación para así facilitar su difusión y discusión. Mientras tanto, la redacción del texto interpretativo avanzaba.

Frente a la interpretación de la apuesta política se ubicó un concepto amplio de política que no se reducía al ámbito estatal ni se reducía a los procesos sociales ya cristalizados, sino que se parte de que la realidad de los sujetos sociales es una realidad estructurada (lo dado) y también estructurándose (lo dándose), es un campo de relaciones y fuerzas en pugna. De allí que se conciba lo político como "la articulación dinámica entre sujetos, prácticas sociales y proyectos, cuyo contenido

11 Para ver las características que conforman el núcleo común de la educación popular, ver Torres (2007). 
específico es la lucha por dar una dirección a la realidad en el marco de opciones viables" (Zemelman, 1989, p. 82-83). Lo político se relaciona con la posibilidad de transformar visiones de futuro en construcciones viables, y esas visiones de futuro van más allá del escenario estatal debido a la existencia de "espacios nuevos de poder que son reflejo de un acelerado proceso de desestatización”(Zemelman, 1989, p. 84), relacionado con las transformaciones del siglo XX señaladas por Sartori (1992) y por el contexto que el neoliberalismo ha creado en nuestra región y la reacción de los sectores populares frente al mismo, como señala Rauber (1995).

Es así que en una concepción amplia de la política, el objetivo final no es (o por lo menos no es suficiente) alcanzar, ocupar o tomar el Estado como el ámbito de acción política por excelencia. La política incluye entonces las pugnas por transformar ámbitos locales y prácticas cotidianas que, en su potencialidad, hacen parte de la lucha por darle dirección a la realidad a partir de una visión de futuro.

A partir de allí se abordó el discurso y las prácticas políticas del proceso. Se identificó que la presencia de un componente utópico ${ }^{12}$ implica que la construcción de la utopía sea un proceso no necesariamente lineal o progresivo en el que inevitablemente se vaya mejorando o creciendo. Por el contrario, la construcción de la utopía parte de un escenario lleno de tensiones y contradicciones en el que tanto las estructuras sociales, como las formas de organización, las formas de relacionarse al interior del colectivo y las prácticas cotidianas son objeto de cuestionamiento, problematización y transformación. Esta idea de la utopía como proceso se expresa en la frase "somos y no somos, estamos siendo", ${ }^{13}$ pues se ubica a la utopía como un horizonte hacia el cual se está caminando, pero que no está construido todavía, sino que está siendo.

La utopía tiene un carácter fuertemente práctico, pues para lograr caminar hacia ese horizonte de futuro se deben definir principios y acciones coherentes para tal fin. Es por esto que Tunjuelo Popular se ve a sí mismo como un proyecto (por definición, en construcción), en donde los principios, las relaciones y las prácticas se encuentran para hacer realizable la utopía.

Para la construcción de la apuesta política se ubica el escenario educativo como un escenario primordial de acción, debido a que allí se reproducen los valores y relaciones sociales que se considera necesario transformar y porque se identifica un potencial de transformación no sólo de las relaciones entre quienes hacen parte de la relación educativa sino en la medida en que allí se potencian transformaciones de orden político amplio. Es decir, las prácticas educativas no son sólo escenarios funcionales al status quo sino que son potencialmente transformadoras.

$\mathrm{Al}$ apostar por la construcción de una educación crítica y transformadora se pueden problematizar los valores y roles (pasividad, individualismo, egoísmo, indiferencia) que reproduce la educación tradicional; se pueden reconocer, valorar, visibilizar y colocar en diálogo saberes que no son reconocidos como válidos; y se puede incidir en la transformación material y concreta del contexto inmediato en

12 La utopía para Zemelman (1989) se entiende como un horizonte de futuro que cumple la función de orientar la construcción de opciones en términos de la disputa política por dar una dirección a la sociedad.

13 Esta frase surgió como reflexión en uno de los grupos focales como reflexión. 
el que se desarrolla, es decir, el territorio en el cual tiene lugar. Esto, pues se ubica una potencialidad trasgresora en la educación popular, enfrentando posiciones como las de Saldaña (2016) en donde no se problematizan las relaciones de poder en la educación; o que afirman que esta debe ser un ámbito en el que se inculque un cumplimiento ciego de las normas, como lo hacen por ejemplo Ripoll, Ripoll y Vásquez (2016); o como lo realizan García, Pulido y Salazar (2016, p. 49) al intentar maquillar la función domesticadora de la autoridad, afirmando que "la autoridad es el ejercicio del cuidado del amor y la disciplina desde los valores y el respeto".

Sin embargo, el escenario educativo, a pesar de sus potencialidades, no es suficiente para lograr la construcción de la utopía. Es decir, la apuesta política debe ir más allá de lo educativo, pues la intención pasa por generar alternativas reales, proyectos de vida colectivos, con anclaje territorial, en donde las personas puedan no solo problematizar las relaciones educativas y las prácticas cotidianas, sino construir unas distintas en coherencia con el proyecto utópico y los principios políticos enunciados previamente.

Este proyecto de vida colectivo que se podría construir alrededor del Rincón Cultural El Caracol se expresa desde la idea de política prefigurativa. Esta idea gramsciana hace referencia a

la construcción, desde el inicio mismo del proceso autonómico, de formas de vinculación, entre nosotros y (a no olvidarlo) con la naturaleza, que prefiguren el horizonte comunista anhelado. Desde esta perspectiva, el fin debería estar, al menos tendencialmente, contenido en los medios mismos. O mejor aún: los medios no serían concebidos como meros medios instrumentalizables, sino que contendrían en su seno, en potencia, los objetivos perseguidos. (Ouviña, 2007, p. 184)

Es necesaria entonces la acción en el presente por transformar la realidad y construir esa utopía que, si bien se ubica en el futuro, debe empezar a materializar opciones en el ahora. Desde esta apuesta no se está esperando a tomar el poder para empezar a agenciar cambios, sino que se busca la construcción anticipada de la sociedad deseada en el presente, guiada por una utopía que, como visión de mundo, espera transformarse en realidad en el futuro.

Por otra parte, en la medida en que las acciones de Tunjuelo Popular visibilizan el carácter político de todos los ámbitos de la vida social como escenarios atravesados por relaciones de opresión y dominación, pero también como potenciales escenarios de emancipación, es que se contribuye a ampliar la esfera de lo político más allá del Estado. Es decir, la apuesta política de Tunjuelo permite reconocer que las relaciones de poder no se concentran solo en el Estado y que los escenarios institucionales-estatales no son los únicos desde los cuáles se puede incidir sobre la realidad.

Además, se identificó una incidencia en la cultura política ${ }^{14}$ en la medida en que se colocan en diálogo valores e ideas críticas alrededor de la educación

14 Entendida desde Gilberto Giménez (1996), citado en Torres (2003, p. 4), como "el conjunto de conocimientos, creencias, valores y actitudes que permiten a los individuos dar sentido a la experiencia rutinaria de sus relaciones con el poder, así como los grupos que le sirven como referencia identitaria”. 
tradicional y se reconoce su carácter político; además, se expresan representaciones críticas frente al Estado y en general el status quo; y se propone la construcción de un proyecto de vida que cuestiona las actitudes y prácticas cotidianas predominantes de las personas respecto al poder y a la autoridad.

Frente a la interpretación de la apuesta educativa, se identificó que para Tunjuelo Popular la educación popular posibilita la confrontación, el cuestionamiento y la problematización de la realidad e incentiva valores como la desobediencia, la solidaridad, la cooperación, el apoyo mutuo o la importancia del diálogo para la construcción de relaciones sociales democráticas. En esa medida, la apuesta educativa contribuye a la construcción de un proyecto de vida colectivo que impulse la emergencia de una sociedad anticapitalista, antipatriarcal y ecologista.

En términos del proceso investigativo en sí, se pudo evidenciar que entre más se concentraran tareas en mí y entre más creciera el texto conjunto que se iba produciendo de la sistematización, la retroalimentación y discusión colectiva era cada vez menor. Al respecto, Alfonso Torres menciona, a partir de una experiencia propia:

La fase interpretativa generó menos participación. Luego de un primer impulso en el cual se involucraron activamente algunos dirigentes, en la medida en que el trabajo requería procesos más sistemáticos de análisis y conceptualización la participación disminuyó y la redacción de los informes de síntesis interpretativa recayó en unos pocos. Ello plantea a la investigación participativa el desafío de ampliar las estrategias de teorización de los procesos reconstruidos, valorar más las reflexiones de los participantes no mediadas por teorías, e incorporar otras narrativas en la síntesis de los resultados de los estudios. (Torres, 2011, p. 53)

Esto señala la necesidad de que una investigación que se plantee a sí misma como participativa desarrolle un proceso formativo no solo en la etapa previa a la investigación, sino que sea un ejercicio permanente y transversal en todas las fases o momentos de su desarrollo. Allí también es vital la incorporación de la cotidianidad dentro de los procesos investigativos con organizaciones populares, las acciones que rompen el ambiente serio de la investigación como cocinar o jugar expresan la necesidad de hacer tanto de la militancia como de los escenarios formativos o investigativos escenarios seriamente alegres y alegremente serios como diría Mariano Algava (2006). La emotividad y la motivación entran a jugar tanto en el proceso de enamorarse de la apuesta política de una organización como del desarrollo de un proceso investigativo. El rescatar la emocionalidad de los procesos sociales e investigativos posibilita que la investigación no se vea como un proceso serio o académico, aparte de las actividades prácticas de la organización, sino que posibilita que se incorpore la práctica investigativa a la cotidianidad de la organización.

Como balance retrospectivo se identifica una falencia en términos de que la forma como se socializaba la información producida en la sistematización 
(con excepción de algunas socializaciones presenciales) era vía correo electrónico. Los antecedentes de baja asistencia a las actividades de la sistematización contribuyeron a fortalecer la sensación de quienes presentaban algún interés por la investigación eran quienes iban a estar. En ese sentido se identifica que se otorgó una confianza excesiva a los medios electrónicos, lo que implicó que se dejaran de intentar otros caminos para garantizar una comunicación y participación efectivas.

Esto dificultó la apropiación de herramientas metodológicas, analíticas e interpretativas por parte de las y los propios integrantes de la experiencia, y tiene como consecuencia que la interpretación de la experiencia responda en su totalidad a una interpretación personal del investigador y no se hayan podido involucrar otras voces y miradas en este momento de la investigación que es donde se complejiza la lectura que se da sobre la propia experiencia. Esto es tanto un llamado de atención para quien realizó en su mayoría la investigación como para el propio colectivo, pues si bien hubo falencias por parte de quien investigó, estas, combinadas con el desinterés que se percibió frente a la sistematización y el incumplimiento de tareas $\mathrm{y}$ acuerdos, se alimentaban mutuamente para generar que el ejercicio investigativo se concentrara cada vez más.

\section{MOMENTO PROSPECTIVO}

Finalizando la investigación, se convocó una reunión para socializar el momento interpretativo y realizar el ejercicio prospectivo. Los aprendizajes que surgieron permiten identificar que la pertenencia y compromiso que se desarrollan en las personas que hacen parte de una organización social tienen un componente afectivo, de allí la importancia de cultivar y fortalecer estos vínculos entre los integrantes de Tunjuelo para así potenciar el involucramiento y la participación en las acciones del proceso. A partir de allí, y del fortalecimiento de vías de comunicación interna, se podría desarrollar una identificación tanto con la apuesta política como la apuesta educativa, posibilitar una sensación y materialización de inclusión de los y las integrantes tanto en las decisiones como en las prácticas del colectivo. Los espacios de acción y encuentro en la dimensión micro, son el punto de partida para fortalecer las otras dimensiones e instancias de Tunjuelo.

\section{REFLEXIONES FINALES}

Un primer gran elemento que se identifica es que las dinámicas internas de la organización afectan directamente las dinámicas investigativas. Es decir, las relaciones de poder al interior de la organización social que está siendo objeto de sistematización van a marcar el ritmo de la investigación, por ejemplo en términos del nivel de participación: si en las actividades propias del proceso hay una participación reducida, es difícil esperar otro panorama cuando se trate del ejercicio investigativo. Esta constatación puede parecer obvia, pero adquiere mucha más relevancia cuando una investigación se plantea de carácter participativo, pues necesita 
de un mayor involucramiento por parte de los sujetos de base de las experiencias asociativas (que no serían sólo fuentes de información).

$\mathrm{Al}$ respecto hay que señalar un elemento específico de los tiempos de Tunjuelo Popular para comprender esta conexión entre ritmos internos e investigativos. Cada año se puede dividir en dos según las actividades realizadas: en la primera mitad se desarrollan las sesiones del proceso educativo popular (el momento de trabajo fuerte), y en la segunda mitad se desarrollan algunas actividades de formación y acompañamiento a los otros procesos de la CPEPEL (este momento se caracteriza por una mayor dispersión en el trabajo). La mayoría de la recolección de la información se realizó en el segundo momento del año, precisamente cuando la participación es menor, lo que claramente incidió en la cantidad de personas que hicieron parte de los grupos focales y las entrevistas. Esto provocó que la investigación gradual pero constantemente se fuera concentrando en mí y que se tuviera la sensación de que la sistematización era una tarea individual y no un ejercicio colectivo, proyectando una distancia entre "el encargado" de la sistematización y el resto. Esto se ve expresado cuando se realizaban aportes o sugerencias de cambios de algún texto por parte de integrantes de Tunjuelo que, a pesar de que estaban encaminados a precisar y mejorar los productos escritos de la investigación, no se tradujo en una intención de discutir colectivamente los contenidos o realizar ejercicios de redacción a varias manos (frente a esto último solo se recibió un aporte).

Otro elemento que atravesó la investigación se relaciona con una tensión entre los tiempos (inflexibles) de la academia y los tiempos propios de la organización articulados con el desarrollo de la sistematización, expresada en el incumplimiento de los acuerdos (relacionado con la concentración de tareas) y la falta de compromiso frente al proceso de la sistematización al interior del colectivo. Esta relación entre la lógica popular y la lógica académica reafirmó la necesidad de potenciar los ejercicios investigativos y de producción de conocimiento en los procesos sociales que le apuestan por una transformación del orden social dominante. No sólo en términos de que lo que se hace y las posibles innovaciones se puedan perder si no son objeto de reflexión, sino en tanto estos ejercicios permiten agenciar cambios al interior de las mismas organizaciones que les permitan cualificar sus propuestas. Además, ejercicios investigativos como el acá presentado invitan a que los sectores populares (y la producción de conocimiento que se realiza desde ellos) disputen el campo académico de validación de saberes y exijan su lugar como sujetos productores de conocimiento legítimo.

Podría decirse que en la investigación se hizo poco pero mucho en la medida en que, si bien se identificaron errores y aspectos para mejorar — pues sigue siendo un trabajo inconcluso - , dentro de Tunjuelo se ha percibido como un ejercicio valioso que es necesario apropiar y continuar, y de a poco se va convirtiendo en un texto de referencia para el mismo proceso y para otras experiencias similares, lo que denota la importancia de sistematizar las experiencias de colectivos y organizaciones populares. 
Quedan también preguntas producto de la misma investigación, entre ellas saber cómo incidió el proceso educativo en las y los educandos después de terminado el ciclo de preuniversitario o qué sensaciones y pensamientos atravesaron a las y los integrantes de Tunjuelo a propósito de la sistematización de su propia experiencia (este aspecto no fue contemplado y podría arrojar información útil para mejorar el proceso investigativo y otros similares). Así mismo queda la necesidad de indagar con mayor profundidad alrededor de las prácticas educativas y, en esa medida, se señala la importancia de llevar un registro sistemático del proceso por varias razones. Por un lado, sirve para poder ir acumulando lo que se hace para que, cuando se incorporen nuevas personas, haya un compendio de las actividades desarrolladas que pueda ser objeto de transformación, reflexión y consolidación. Esto implicaría la necesidad de crear espacios de formación tanto políticos como educativos para tener elementos que permitan realizar una lectura crítica de las actividades desarrolladas y que se dé un ejercicio de alejamiento de la propia experiencia para analizarla sin que el involucramiento emocional sea tan fuerte. Por otro lado, y mucho más importante, se posibilitaría una reflexión autocrítica de la práctica educativa que permita ampliar el sentido educativo y pedagógico de la misma, y en esa medida se potencie la cualificación de una práctica concreta de educación popular.

\section{REFERENCIAS}

ALGAVA, M. Jugar y jugarse. Buenos Aires: Ediciones América Libre, 2006.

CENDALES, L. La metodología de la sistematización: una construcción colectiva. Revista Aportes, Bogotá, n. 57, p. 91-114, jun. 2004.

CENDALES, L.; TORRES, A. La sistematización como experiencia investigativa y formativa. Revista La Piragua, Ciudad de Panamá, n. 23, p. 29-38, 2006.

DE SOUSA SANTOS, B. De la mano de Alicia. Lo social y lo político en la postmodernidad. Bogotá: Siglo del Hombre; Uniandes, 1998.

FALS BORDA, O. Orígenes universales y retos actuales de la IAP. Revista Análisis Político, Bogotá, n. 38, p. 73-89, sept./dic. 1999.

GARCÍA, M.; PULIDO, C.; SALAZAR, M. Sin tablero: aula para la emancipación del maestro. Revista Inclusión \& Desarrollo, Bogotá, n. 1, v. 3, p. 47-55,jul./dic. 2016. Disponible en: http://dx.doi.org/10.26620/uniminuto.inclusion.3.2.2016.47-55. Acceso en: 17 abr. 2018.

GHISO, A. De la práctica singular al diálogo con lo plural. Aproximaciones a otros tránsitos y sentidos de la sistematización en épocas de globalización. Medellín: FUNLAM, ago. 1998. Disponible en: http://unpan1.un.org/intradoc/groups/public/ documents/icap/unpan033101.pdf. Acceso en: 15 oct. 2019.

GIMÉNEZ, G. Cultura política e identidad. San Andrés de Totoltepec: documento inédito, 1996.

GÓMEZ, S. Concepto de la tesis de maestría. Comunicación personal, 2017. 
GÓMEZ, S.; MEDINA, J.; RIAÑO, H. Sistematización de la experiencia del Movimiento Alimentario de Bosa - MAB. 2012.318 p. Tesis (Maestría en Desarrollo Educativo y Social) - Fundación Centro Internacional de Educación y Desarrollo Humano y Universidad Pedagógica Nacional, Bogotá, 2012.

MANTILLA, I. Cómo fue la admisión a la Universidad Nacional. El Espectador, Bogotá, 12 oct. 2016. Disponible en: https://www.elespectador.com/opinion/opinion/como-fuela-admision-la-universidad-nacional-columna-660458. Acceso en: 25 sept. 2018.

MARIÑO, G. Sistematizando la sistematización. Revista Aportes, Bogotá, n. 57, p. 43-52, jun. 2004.

MARIÑO, G. Sistematización de experiencias. Una propuesta desde la educación popular. 2011. Disponible en: http://www.germanmarino.com/phocadownloadpap/ Sistematizacin\%20sinopsis.pdf. Acceso en: 12 marzo 2018.

MARTÍNEZ-GÓMEZ, N. Del río al caracol: transformando la cotidianidad. Sistematización de la experiencia de la Pre-Universidad Tunjuelo Popular (2012-2016). 2017.265 p.Tesis (Maestría en Estudios Sociales) — Universidad Pedagógica Nacional, Bogotá, 2017.

MEJÍA, M. Diálogo-confrontación de saberes y negociación cultural. Ejes de las pedagogías de la educación popular: una construcción desde el sur. In: CENDALES, L.; MEJÍA, M.; MUÑOZ, J. (Eds.). Pedagogías y metodologías de la educación popular “Se hace camino al andar". Bogotá: Ediciones Desde Abajo, 2016. p. 227-249. MENDOZA, N.; TORRES, A. La sistematización de experiencias en educación popular. In: CENDALES, L.; MEJÍA, M.; MUÑOZ, J. (Eds.). Entretejidos de la educación popular en Colombia. Bogotá: Ediciones desde abajo, 2013. p. 155-184.

MÚNERA, L. Crítica y ciencias sociales. In: MÚNERA, L. (Comp.). Ensayos críticos de teoría política. Bogotá: Universidad Nacional de Colombia, 2009. p. 15-36.

OUVIÑA, H. Hacia una política prefigurativa. Algunos recorridos e hipótesis en torno a la construcción del poder popular. In: MAZZEO, M. et al. Reflexiones sobre el poder popular. Buenos Aires: Editorial E1 Colectivo, 2007. p. 163-192.

RAUBER, I. Actores sociales, luchas reivindicativas y política popular. Revista Pasos, San José, n. 62, p. 21-45, nov./dic. 1995.

REVISTA INTERNACIONAL MAGISTERIO. Editorial. Revista Internacional Magisterio, Bogotá, n. 33, p. 6, jul. 2008.

RIPOLL, M.; RIPOLL, A.; VÁSQUEZ, F. La formación ciudadana en la escuela: educandos críticos y activos. Revista Inclusión \& Desarrollo, Bogotá, n. 1, v. 3, p. 24-33, jul./dic. 2016. Disponible en: http://dx.doi.org/10.26620/uniminuto. inclusion.3.2.2016.24-33. Acceso en: 17 abr. 2018.

SALDAÑA, R. Incidencias de las transformaciones sociales en lo curricular. Revista Inclusión \& Desarrollo, Bogotá, n. 1, v.3, p. 58-66,jul./dic. 2016. Disponible en: http:// dx.doi.org/10.26620/uniminuto.inclusion.3.2.2016.58-66. Acceso en: 17 abr. 2018.

SARTORI, G. Política. In: SARTORI, G. Elementos de teoría política. Madrid: Alianza Editorial, 1992. p. 205-221. 
TORRES, A. La política de las organizaciones populares. In: TORRES, A.; MENDOZA, N.; VARGAS, A.; GONZÁLEZ, M.; AVENDAÑO, M.; VALLEJO, M. Organizaciones populares, identidades colectivas y ciudadanía en Bogotá. Bogotá: Universidad Pedagógica Nacional, 2003. p. 155-208.

TORRES, A. La educación popular. Trayectoria y actualidad. Bogotá: Editorial El Búho, 2007.

TORRES, A. La interpretación en la sistematización de experiencias. Decisio, Ciudad de México, n. 28, p. 47-55, enero/abr. 2011. Disponible en: https://cdn.crefal.org/ CREFAL/revistas-decisio/decisio28.pdf. Acceso en: 21 marzo 2018.

VASCO, C. Sistematizar o no, he ahí el problema. Revista Internacional Magisterio, Bogotá, n. 33, p. 20-33, jul. 2008.

ZEMELMAN, H. De la historia a la política. La experiencia de América Latina. México: Siglo XXI Editores, 1989.

\section{SOBRE EL AUTOR}

Nicolás Martínez-Gómez es magíster en estudios sociales por la Universidad Pedagógica Nacional (Colombia). Profesor de la Corporación Universitaria Minuto de Dios (Colombia).

E-mail:nmartinezgo@yahoo.com

Recibido el 9 de noviembre de 2018 Aprobado el 30 de julio de 2019 